\title{
Incidencia Del Uso Del Podcast Como Estrategia De Aprendizaje Para La Comprensión Auditiva Del Inglés
}

\author{
Edison Renato Ruiz López \\ Patricia Pilar Moyota Amaguaya
}

Magíster en Lingüística y didáctica de la enseñanza de Idiomas Extranjeros Docente del Centro de Idiomas de la Escuela Superior Politécnica de

Chimborazo, Ecuador

Porras Pumalema Sandra Paulina

Magíster en Desarrollo de la Inteligencia y Educación

Docente del Centro de Idiomas de la Escuela Superior Politécnica de

Chimborazo, Ecuador

Rodríguez Durán María Eugenia

Magister en la Enseñanza del Idioma Inglés como lengua extranjera

Docente del Centro de Idiomas de la Escuela Superior Politécnica de

Chimborazo, Ecuador

Rodríguez Arellano Nancy Georgina

Magister en Docencia Universitaria y Administración Educativa

Docente del Centro de Idiomas de la Escuela Superior Politécnica de

Chimborazo, Ecuador

Doi:10.19044/esj.2019.v15n2p27 ～URL:http://dx.doi.org/10.19044/esj.2019.v15n2p27

Resumen

Esta investigación tuvo como objetivo analizar el uso del podcast como estrategia de aprendizaje con dispositivos móviles y determinar su incidencia en la comprensión auditiva del idioma inglés. Para ello, se consideró un diagnóstico previo que permitió elaborar un rediseño curricular, el cual contempló un plan de intervención estructurado por un conjunto de actividades que fueron aplicadas a 67 estudiantes del Segundo Año de Bachillerato General Unificado de la Unidad Educativa Pedro Vicente Maldonado durante el año lectivo 2016-2017. La investigación fue cuasi experimental, bibliográfica, relacional, hipotética deductiva y de campo, donde se estableció una relación causa efecto entre las variables intervinientes. La muestra está representada por un grupo de control conformado por 31 estudiantes, que desarrollaron las actividades mediante el método tradicional; y un grupo experimental de 36 estudiantes quienes utilizaron el podcast a través de 
dispositivos móviles. Los dos grupos fueron evaluados en dos momentos (antes y después), mediante un examen internacional (PET) de Cambridge. Los datos fueron tabulados, analizados e interpretados a través del cálculo del puntaje $\mathrm{Z}$. La misma que determinó, que el uso de podcast a través de dispositivos móviles y aplicado como estrategia de aprendizaje, incidió positivamente en la comprensión auditiva del idioma inglés.

Palabras Claves: Podcast, estrategia, incidencia, comprensión auditiva, dispositivos móviles

\title{
Incidence of the Use of Podcast as a Learning Strategy for the Auditory Understanding of English
}

\author{
Edison Renato Ruiz López \\ Patricia Pilar Moyota Amaguaya
}

Magíster en Lingüística y didáctica de la enseñanza de Idiomas Extranjeros Docente del Centro de Idiomas de la Escuela Superior Politécnica de

Chimborazo, Ecuador

Porras Pumalema Sandra Paulina

Magíster en Desarrollo de la Inteligencia y Educación

Docente del Centro de Idiomas de la Escuela Superior Politécnica de

Chimborazo, Ecuador

Rodríguez Durán María Eugenia

Magister en la Enseñanza del Idioma Inglés como lengua extranjera

Docente del Centro de Idiomas de la Escuela Superior Politécnica de

Chimborazo, Ecuador

Rodríguez Arellano Nancy Georgina

Magister en Docencia Universitaria y Administración Educativa

Docente del Centro de Idiomas de la Escuela Superior Politécnica de

Chimborazo, Ecuador

\begin{abstract}
This paper focuses on analyzing the use of podcast as a learning strategy applied to mobile devices and it aims to determine its impact on English Language's listening comprehension. In doing so, a prior diagnosis was considered. This diagnosis allows the development of a curriculum redesign which comprises of an interventional plan structured by a set of
\end{abstract}


activities implemented on 67 students of the second year of General Unified Baccalaureate at Pedro Vicente Maldonado Educational Unit during the academic period 2016 - 2017. The research was quasi-experimental, bibliographic, relational, hypothetical deductive and a field study, where a causal relationship between the intervening variables was established. The sample is represented by a control group made up of 31 students who developed the activities using the traditional method; and an experimental group of 36 students who used the podcast through mobile devices. Both groups were evaluated in two moments (before and after) through the Preliminary English Test (PET) from Cambridge. The data was tabulated, analyzed, and interpreted through the $\mathrm{Z}$ score calculation. It shows that the use of podcast through mobile devices, and which is implemented as a learning strategy, had a significant positive impact on English listening comprehension.

Keywords: Podcast, Strategies, incidence, listening Comprehension, mobile devices

\section{INTRODUCCIÓN}

A través de la presente investigación se realizó un análisis de la implementación del aprendizaje electrónico móvil a través del podcast como estrategia para mejorar la comprensión auditiva del inglés delos estudiantes de segundo año de bachillerato de la Unidad Educativa Pedro Vicente Maldonado de la ciudad de Riobamba.

En la investigación se establece la importancia de utilizar los recursos didácticos y los insumos tecnológicos que presenta el mundo actual dentro y fuera de las aulas de clase como parte del proceso de enseñanza-aprendizaje del idioma inglés, fomentando el autoaprendizaje y sobrepasando las barreras impuestas por el sistema educativo tradicional para de esta manera mejorar el rendimiento de los estudiantes.

A diferencia de la aplicación de métodos retrógradas y aburridos que salen absolutamente del contexto actual del mundo real, quienes han tenido problemas de aprendizaje del idioma inglés están al tanto de que actualmente el internet ha permitido experimentar con herramientas y aplicaciones web para superar dichas frustraciones Partiendo de la dificultad que tienen los estudiantes al momento de escuchar una pregunta simple, un diálogo, o una conversación en inglés, es decir, en comprender el idioma de forma oral, la presente investigación pretende determinar cómo la utilización del Aprendizaje electrónico móvil a través del podcast puede incidir en la comprensión del inglés.

El podcast, al ser una herramienta auditiva, permite desarrollar las destrezas auditivas del idioma inglés a través de interesantes opciones como cuentos, noticias, canciones, etc., y de igual manera ayudará a los estudiantes 
a mantenerse interesados en la temática propuesta por el docente en el contenido de dicha herramienta.

En la Universidad de Austin en Texas (2013) sostienen que "un podcast es un audio digital o un archivo de video que es episódico, descargable, que corre en algún programa, principalmente con un conductor o tema, y normalmente vía un alimentador automático con un software computacional".

El podcast es un archivo sonoro que podemos descargar de Internet de forma automática mediante un sistema de suscripción, y sincronizarlo con un reproductor digital portátil. Una vez que el usuario ha recibido el podcast al que está suscrito, puede escucharlo en varias ocasiones. El oyente no solo decide el cuándo, sino que puede parar en todo momento la audición o bien avanzar y retroceder en el archivo de audio (Sellas \& Tony, 2011).

El uso didáctico del podcast enriquece la experiencia de aprendizaje (Boulos, Maramba \& Wheeler, 2006), potencia la atención del estudiante y su reflexión (Baird \& Fisher, 2006), incrementa su satisfacción (Miller \& Piller, 2005) e, inclusive, reduce la ansiedad (Chan \& Lee, 2005). En lo que respecta al m-learning existen diversas definiciones. Kinshuk (2003) lo define como la evolución del aprendizaje electrónico, como una tendencia producto de la propagación de los sistemas de comunicación actuales, mientras que Quinn (2001) lo visualiza como la intersección entre computación móvil y aprendizaje electrónico, con accesibilidad a los recursos requeridos y soporte de aprendizaje efectivo.

En la teoría cognitiva, la comprensión auditiva del lenguaje es vista como un proceso activo, en el cual el significado de un contenido se construye a través de una compleja interacción entre las características del hablante, los tipos de conocimiento declarativo a los que se accede y el uso de procesos estratégicos para mejorar la comprensión (Chamot, 1995, p.16).

De acuerdo con Goss (1982), la compresión auditiva es considerada como un sistema de procesamiento de la información. Tal procesamiento consiste en la manera en que se accede a la información, se almacena y se recupera en un intento de dar sentido al mundo que nos rodea.

Igualmente, la comprensión auditiva es una de las destrezas lingüísticas de cualquier idioma, la cual se refiere a la interpretación del discurso oral. En ella intervienen, además del componente estrictamente lingüístico, factores cognitivos, perceptivos, de actitud y sociológicos.

Es también una capacidad comunicativa que abarca el proceso completo de interpretación del discurso, desde la descodificación y comprensión lingüística de la cadena fónica (fonemas, sílabas, palabras, etc.) hasta la interpretación y la valoración personal, de modo que, a pesar de su carácter receptivo, requiere una participación activa del oyente. 
La comprensión auditiva está relacionada con la comprensión lectora; sin embargo, se trata de dos destrezas tan distintas como distintas son la lengua oral y la lengua escrita.

La Asociación Internacional de Escucha (ILA en inglés) (1996), define esta habilidad como el proceso de recepción, la construcción de significado a partir de la recepción de mensajes y responder a estos mensajes hablados y /o no verbales. No obstante, se podría entender que el ser humano está en constante percepción auditiva porque no podemos cerrar físicamente ese canal, sin embargo, sería fácil asumir que estamos siempre en un estado de escucha cuando únicamente estamos oyendo.

Como lo señala Boquete (2011) la destreza auditiva es un hecho que persigue un objetivo concreto que es el de recibir información y dar una respuesta a esa información en base a entender algo, por lo que es primordial un proceso de compresión del mensaje. La comprensión auditiva comprende:

- La lengua básica

- La información clave en situaciones diversas como apoyo contextual

- La lengua en intercambios sociales y necesita ayuda contextual en los intercambios académicos

- La lengua en la mayoría de los contextos

La comprensión auditiva es una destreza, es decir, es una habilidad lingüística que el estudiante puede desarrollar. En este sentido, es necesario recordar que la comprensión de un mensaje transmitido oralmente lejos está de ser un proceso de recepción pasiva. La clasificación de las destrezas en destrezas activas (escribir y hablar) y destrezas pasivas (leer y escuchar) no responde a la realidad de ningún hablante. En efecto, la comprensión auditiva es una destreza activa que consiste "en desarrollar una actividad de interpretación que tiene sus propias técnicas y estrategias" (Peris, 1996).

Por tal motivo, las herramientas tecnológicas y nuevas tecnologías de la información (TIC) son aplicadas en el desarrollo de la compresión auditiva y utilizadas por los estudiantes del Segundo Año de Bachillerato de la Unidad Educativa Pedro Vicente Maldonado. De ahí la importancia del podcast como recurso adicional en la enseñanza del inglés, en pocas palabras, para que los docentes del área de lengua extranjera puedan reforzar los conocimientos de los estudiantes y desarrollar su destreza auditiva.

Por lo antes mencionado, esta investigación considera importante determinar si el uso de podcast como estrategia de aprendizaje para el desarrollo de la comprensión auditiva de inglés incide positivamente. Para ello, se utilizarán dispositivos móviles como herramienta tecnológica de aprendizaje. 


\section{METODOLOGÍA}

La modalidad de la investigación fue socioeducativa orientada a la producción de conocimiento, para tratar de añadir a la educación tradicional métodos de enseñanza - aprendizaje tecnológicos acordes a la realidad educacional actual.

La investigación contribuyó al conocimiento de la realidad social y a la búsqueda de soluciones idóneas a las necesidades existentes tanto de docentes como estudiantes. Para tal fin fue necesario profundizar en el conocimiento de los estudiantes de Segundo Año de Bachillerato de la Unidad Educativa Pedro Vicente Maldonado mediante el diseño de un programa de intervención que favoreció la capacidad de adaptación y participación social de los estudiantes a las nuevas tecnologías de la información. Además, se favoreció la transmisión del conocimiento generado en el desarrollo de la actividad docente e investigadora del equipo.

La investigación se basó en un enfoque cuali-cuantitativo ya que se realizó un análisis bibliográfico, lo cual permitió obtener referencias investigativas acerca del aprendizaje electrónico móvil a través del podcast) realizadas a nivel local e internacional. El proceso de recolección de información fue útil para la construcción del objeto de la investigación bibliográfica y documental, garantizando así la calidad de los fundamentos teóricos de la investigación.

El estudio conjugó el análisis interpretativo de resultados en base a una medición numérica y el análisis estadístico de respuestas (previo y posteriormente) mediante un test aplicado a la población investigada. Para ello, se recurrió a la vía hipotético-deductiva a fin de comprobar los objetivos planteados en cuanto al uso de del podcast y la comprensión auditiva del inglés.

La investigación recabó información de los estudiantes para analizar la comprensión auditiva del idioma inglés posterior al uso del podcast como herramienta de refuerzo en el proceso de enseñanza aprendizaje. Para ello se utilizaron métodos cualitativos (encuesta) y el respectivo análisis bibliográfico, métodos que interactuaron con los datos cuantitativos (resultados estadísticos de los test aplicados) y se apoyaron mutuamente en la dilucidación del problema.

El diseño de la selección de los sujetos de estudio no es al azar, pues se consideró la efectividad del uso del aprendizaje electrónico móvil a través del podcast) para la compresión auditiva del idioma inglés en los estudiantes de Segundo Año de Bachillerato General Unificado "P" de la Unidad Educativa Pedro Vicente Maldonado durante el año lectivo 2016-2017.

La investigación fue cuasi-experimental y se llevó a cabo con grupos de trabajo equivalentes (experimental y grupo de control) que fueron sometidos a pre-test y post-test para una posterior comparación. 
Antes de iniciar la intervención se aplicó una prueba estandarizada en los grupos experimental y de control. Posterior a esto se llevó a cabo la intervención y al final de la misma se aplicó el mismo instrumento a manera de post test para poder determinar el nivel de influencia entre las variables y los resultados de la intervención.

La población está conformada por 67 estudiantes de Segundo Año de Bachillerato General Unificado "P" de la Unidad Educativa Pedro Vicente Maldonado durante el año lectivo 2016-2017. Se ha elegido este grupo de estudiantes a elección del investigador estableciendo dos grupos: uno de control y otro experimental.

El grupo de control está compuesto por 31 estudiantes de Segundo Año y el grupo experimental está compuesto por otros 36 estudiantes del mismo año y de la misma institución.

La intervención que se aplicó para mejorar la comprensión auditiva del inglés en los estudiantes, consistió en la aplicación de la estrategia del uso del aprendizaje electrónico móvil a través del podcast previo a la práctica de ejercicios de escucha (listening exercises) en el idioma inglés, y tuvo una duración de 8 semanas.

La investigación fue estructurada correlacional bivariada (con variables de causa y efecto) con intervención, por lo que se constituyó en un proyecto de investigación - acción. La correlación fue establecida entre la implementación del aprendizaje electrónico móvil a través del uso del podcast y la comprensión auditiva del idioma inglés, las cuales son las variables de estudio. Esta correlación se hizo en base a la medición cualitativa y cuantitativa de las variables mencionadas.

Las técnicas empleadas en la presente investigación fueron la observación directa y la encuesta. Se utilizaron métodos teóricos, el análisis y la síntesis para procesar la información teórica y para sistematizar los datos que se recogieron en el campo en el momento en el que se construyó el marco teórico y en el momento en el que los datos recopilados en el experimento fueron analizados.

El método empírico con el que se recabó la información en este caso fue la técnica de la encuesta con su respectivo cuestionario. Los ítems están desarrollados de acuerdo a la realidad objeto de estudio, esto con el objetivo de recolectar datos para la investigación sobre la implementación del aprendizaje electrónico móvil a través del podcast en la comprensión auditiva del idioma inglés y conocer cómo los docentes desarrollan sus clases de inglés para así determinar la viabilidad del objetivo general de la investigación. Con los resultados arrojados luego de la aplicación de la encuesta, será posible complementar la información sobre las variables utilizadas en la investigación.

Para medir los conocimientos que tienen los y las estudiantes de la población de muestra acerca del uso del podcast para trabajar la comprensión 
auditiva del inglés se utilizó una prueba estandarizada internacional de Cambridge - examen de escucha (listening test), para lo cual se contó con la debida autorización de las autoridades de la Unidad Educativa Pedro Vicente Maldonado. Al haber utilizado la prueba estandarizada internacional de Cambridge - examen de escucha (listening test) como pre y post test, este instrumento no necesitó validación; sin embargo, el formato de encuesta utilizado fue consultado con docentes expertos en elaboración de instrumentos relacionados a la asignatura.

Para la validación del instrumento, se entregó a los profesionales los documentos que se detallan a continuación :

1. Carta de presentación

2. Documentos con el objetivo general y objetivos específicos de la investigación

3. Documento con el objetivo del instrumento

4. El cuestionario de la encuesta

5. La operacionalización de las variables de la investigación

Se utilizaron técnicas lógicas y estadísticas de programas como Microsoft Excel, para la elaboración de gráficos y tablas que permitieron ordenar los datos para una mejor interpretación de los mismos.

Para el orden e interpretación de variables en escala nominal se utilizaron números y frecuencias, $\mathrm{y}$ para variables de escala continua se utilizaron medidas de tendencia central y dispersión. De igual forma, se realizó un análisis bivariable para establecer relaciones entre variables.

Para analizar la información recopilada a través de los instrumentos se usó estadística inferencial, y por medio de la inducción se determinaron propiedades de una población estadística a partir de una pequeña parte de la misma. Su objetivo fue obtener conclusiones útiles para hacer deducciones sobre una totalidad, basándose en la información numérica obtenida.

Al ser una investigación cuanti-cualitativa basada en el análisis bibliográfico y estadístico, el proceso fue el siguiente:

1. Vaciado de datos de los instrumentos en el computador

2. Tabulación de datos

3. Elaboración de gráficos estadísticos

4. Análisis cuanti-cualitativo de los datos

5. Conclusiones y recomendaciones

\section{RESULTADOS}

\section{Pre test}

Pre Test Grupo de Control

De las distribuciones normales tabulamos los siguientes datos obtenidos de la lista de cotejo aplicada a los estudiantes, donde:

$x:$

Calificaciones obtenidas 
fi: $\quad$ Frecuencia: Número de estudiantes que obtuvieron esa calificación (x)

$f(x i) \quad$ Producto entre la frecuencia (fi) y el número de calificaciones obtenidas (x).

$x^{\wedge}$ 2: $\quad$ Cuadrado del número de calificaciones $(\mathrm{x})$.

fi $\left(x^{\wedge} 2\right)$ : $\quad$ Producto entre la frecuencia y el número de calificaciones al cuadrado $\left(x^{\wedge} 2\right)$

fr: $\quad$ Frecuencia relativa

$X: \quad$ Media aritmética $\left(\sum \mathrm{fi} / \sum \mathrm{xifi}\right)$

$s: \quad$ Desviación estándar $\left(\sum\right.$ fixi $\left.2 / \sum \mathrm{fi}\right)-\mathrm{X}^{\wedge} 2$

Tabla 1. Pre Test Grupo de Control

\begin{tabular}{|c|c|c|c|c|c|}
\hline Calificación & $\begin{array}{c}\text { Frecuencia } \\
\text { Absoluta }\end{array}$ & Producto & & & $\begin{array}{c}\text { Frecuencia } \\
\text { Relativa }\end{array}$ \\
\hline $\mathrm{xi}$ & $\mathrm{Fi}$ & $\mathrm{xifi}$ & $\mathrm{xi} 2$ & fixi2 & \\
\hline 1 & 0 & 0 & 1 & 0 & 0 \\
\hline 2 & 0 & 0 & 4 & 0 & 0 \\
\hline 3 & 4 & 12 & 9 & 36 & 0,12903226 \\
\hline 4 & 5 & 20 & 16 & 80 & 0,16129032 \\
\hline 5 & 7 & 35 & 25 & 175 & 0,22580645 \\
\hline 6 & 2 & 12 & 36 & 72 & 0,06451613 \\
\hline 7 & 6 & 42 & 49 & 294 & 0,19354839 \\
\hline 8 & 4 & 32 & 64 & 256 & 0,12903226 \\
\hline 9 & 3 & 27 & 81 & 243 & 0,09677419 \\
\hline 10 & 0 & 0 & 100 & 0 & 0 \\
\hline & $\mathbf{3 1}$ & $\mathbf{1 8 0}$ & $\mathbf{3 8 5}$ & $\mathbf{1 1 5 6}$ & \\
\hline
\end{tabular}

Fuente: Pre Test (Grupo de Control)

Elaborado por: Ruiz Renato, 2018

Media Aritmética: 5,81

Desviación Estándar: 1,88

Tabla 2. Pre Test Grupo Experimental

\begin{tabular}{|c|c|c|c|c|c|}
\hline Calificación & $\begin{array}{c}\text { Frecuencia } \\
\text { Absoluta }\end{array}$ & Producto & & & $\begin{array}{c}\text { Frecuencia } \\
\text { Relativa }\end{array}$ \\
\hline $\mathrm{xi}$ & $\mathrm{Fi}$ & $\mathrm{xifi}$ & $\mathrm{xi} 2$ & fixi2 & \\
\hline 1 & 0 & 0 & 1 & 0 & 0 \\
\hline 2 & 1 & 2 & 4 & 4 & 0,02777778 \\
\hline 3 & 1 & 3 & 9 & 9 & 0,02777778 \\
\hline 4 & 11 & 44 & 16 & 176 & 0,30555556 \\
\hline 5 & 6 & 30 & 25 & 150 & 0,16666667 \\
\hline 6 & 5 & 30 & 36 & 180 & 0,13888889 \\
\hline 7 & 8 & 56 & 49 & 392 & 0,22222222 \\
\hline 8 & 3 & 24 & 64 & 192 & 0,08333333 \\
\hline 9 & 1 & 9 & 81 & 81 & 0,02777778 \\
\hline 10 & 0 & 0 & 100 & 0 & 0 \\
\hline & $\mathbf{3 6}$ & $\mathbf{1 9 8}$ & $\mathbf{3 8 5}$ & $\mathbf{1 1 8 4}$ & \\
\hline
\end{tabular}

Fuente: Pre Test (Grupo de Experimental)

Elaborado por: Ruiz Renato, 2018

Media Aritmética: 5,50

Desviación Estándar: 1,62 
Análisis comparativo Pre Test

Cálculo de la Media Aritmética

\begin{tabular}{|c|c|}
\hline \multicolumn{1}{|c|}{ Grupo de Control } & Grupo Experimental \\
\hline $\mathrm{x}_{\mathrm{c}}=\frac{\sum\left(\mathrm{x}_{\mathrm{i}} \cdot \mathrm{f}_{\mathrm{i}}\right)_{\mathrm{c}}}{\mathrm{n}_{\mathrm{c}}}=\frac{180}{31} \approx 5,81$ & $\mathrm{x}_{\mathrm{e}}=\frac{\sum\left(\mathrm{x}_{\mathrm{i}} \cdot \mathrm{f}_{\mathrm{i}}\right)_{\mathrm{e}}}{\mathrm{n}_{\mathrm{e}}}=\frac{198}{36} \approx 5,50$ \\
\hline
\end{tabular}

Fuente: Pre test - Media Aritmética

Elaborado por: Ruiz, Renato, 2018

Cálculo de la Desviación Estándar

\begin{tabular}{|c|c|}
\hline Grupo de Control & Grupo Experimental \\
\hline$\sigma_{c}=\sqrt{\frac{\sum x_{i}^{2} f_{i}}{n_{c}}-x_{c}}{ }^{2}$ & $\sigma_{\mathrm{e}}=\sqrt{\frac{\sum \mathrm{x}_{\mathrm{i}}^{2} \mathrm{f}_{\mathrm{i}}}{\mathrm{n}_{\mathrm{e}}}-\mathrm{x}_{\mathrm{e}}}{ }^{2}$ \\
\hline$\sigma_{c}=\sqrt{\frac{1156}{31}-5,81^{2}}$ & $\sigma_{e}=\sqrt{\frac{1184}{36}-5,50^{2}}$ \\
\hline$\sigma_{c}=\sqrt{3,575}$ & $\sigma_{e}=\sqrt{2,64}$ \\
\hline$\sigma_{c}=1,88$ & $\sigma_{e}=1,62$ \\
\hline
\end{tabular}

Fuente: Pre test-Desviación estándar

Elaborado por: Ruiz Renato, 2018

\section{Media aritmética - PRE TEST}

\subsection{1}
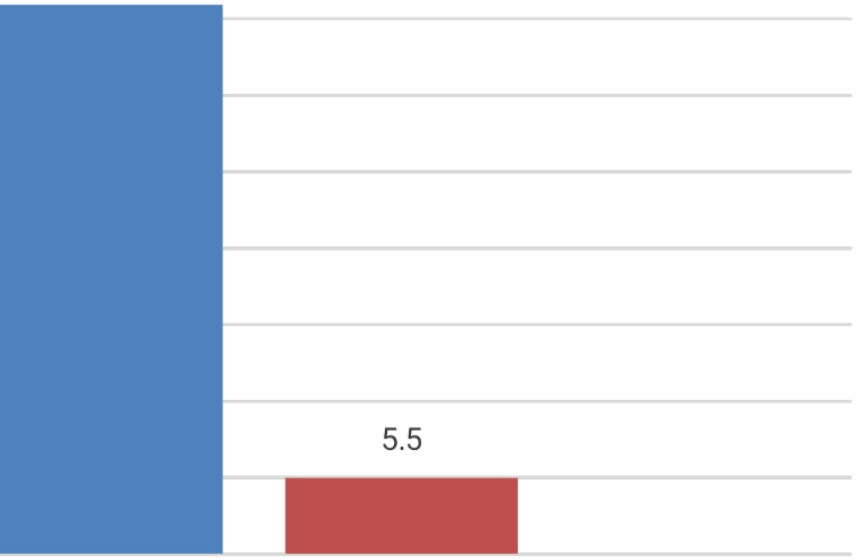

Gráfico 1. Media Aritmética Pre Test (Grupo control vs Grupo experimental) 
La media aritmética del grupo de control es de 5,81 sobre 10 puntos (puntaje total del pre test), mientras que la media del grupo experimental es de 5,50 , lo que significa que ambos grupos tienen una calificación promedio deficiente, insatisfactoria. Por lo tanto, se deduce que el grupo de alumnos de la investigación posee un nivel bastante bajo de comprensión auditiva del idioma inglés

\section{Post Test}

Tabla 2. Post Test Grupo de Control

\begin{tabular}{|c|c|c|c|c|c|}
\hline Calificación & $\begin{array}{c}\text { Frecuencia } \\
\text { Absoluta }\end{array}$ & Producto & & & $\begin{array}{c}\text { Frecuencia } \\
\text { Relativa }\end{array}$ \\
\hline $\mathbf{X i}$ & fi & xifi & xi2 & fixi2 & \\
\hline 1 & 0 & 0 & 1 & 0 & 0 \\
\hline 2 & 1 & 2 & 4 & 4 & 0,032258065 \\
\hline 3 & 0 & 0 & 9 & 0 & 0 \\
\hline 4 & 6 & 24 & 16 & 96 & 0,193548387 \\
\hline 5 & 7 & 35 & 25 & 175 & 0,225806452 \\
\hline 6 & 5 & 30 & 36 & 180 & 0,161290323 \\
\hline 7 & 8 & 56 & 49 & 392 & 0,258064516 \\
\hline 8 & 0 & 0 & 64 & 0 & 0 \\
\hline 9 & 4 & 36 & 81 & 324 & 0,129032258 \\
\hline \multirow[t]{2}{*}{10} & 0 & 0 & 100 & 0 & 0 \\
\hline & 31 & 183 & 385 & 1171 & \\
\hline
\end{tabular}

Fuente: Pre Test

Elaborado por: Renato Ruiz López

Media Aritmética: 5,90

Desviación Estándar: 1,71

Tabla 3. Post Test Grupo Experimental

\begin{tabular}{|c|c|c|c|c|c|}
\hline Calificación & $\begin{array}{c}\text { Frecuencia } \\
\text { Absoluta }\end{array}$ & Producto & & & $\begin{array}{c}\text { Frecuencia } \\
\text { Relativa }\end{array}$ \\
\hline $\mathbf{x i}$ & $\mathbf{f i}$ & $\mathbf{x i f i}$ & $\mathbf{x i 2}$ & $\mathbf{f i x i 2}$ & \\
\hline 1 & 0 & 0 & 1 & 0 & 0 \\
\hline 2 & 0 & 0 & 4 & 0 & 0 \\
\hline 3 & 0 & 0 & 9 & 0 & 0 \\
\hline 4 & 0 & 0 & 16 & 0 & 0 \\
\hline 5 & 1 & 5 & 25 & 25 & 0,02777778 \\
\hline 6 & 1 & 6 & 36 & 36 & 0,02777778 \\
\hline 7 & 2 & 14 & 49 & 98 & 0,05555556 \\
\hline 8 & 16 & 128 & 64 & 1024 & 0,44444444 \\
\hline 9 & 7 & 63 & 81 & 567 & 0,19444444 \\
\hline 10 & 9 & 90 & 100 & 900 & 0 \\
\hline & $\mathbf{3 6}$ & $\mathbf{3 0 6}$ & $\mathbf{3 8 5}$ & $\mathbf{2 6 5 0}$ & \\
\hline
\end{tabular}

Fuente: Post Test

Elaborado por: Renato Ruiz López 
Media Aritmética: 8,50

Desviación Estándar: 1,17

Análisis comparativo Post Test

Cálculo de la Media Aritmética

\begin{tabular}{|c|c|}
\hline Grupo de Control & Grupo Experimental \\
\hline $\mathrm{x}_{\mathrm{c}}=\frac{\sum\left(\mathrm{x}_{\mathrm{i}} \cdot \mathrm{f}_{\mathrm{i}}\right)_{\mathrm{c}}}{\mathrm{n}_{\mathrm{c}}}=\frac{183}{31} \approx 5,90$ & $\mathrm{x}_{\mathrm{e}}=\frac{\sum\left(\mathrm{x}_{\mathrm{i}} \cdot \mathrm{f}_{\mathrm{i}}\right)_{\mathrm{e}}}{\mathrm{n}_{\mathrm{e}}}=\frac{306}{36} \approx 8,50$ \\
\hline
\end{tabular}

Fuente: Poste test - Media Aritmética

Elaborado por: Ruiz Renato, 2018

Cálculo de la Desviación Estándar

\begin{tabular}{|l|l|}
\hline \multicolumn{1}{|c|}{ Grupo de Control } & \multicolumn{1}{c|}{ Grupo Experimental } \\
$\sigma_{\mathrm{c}}=\sqrt{\frac{\sum \mathrm{x}_{\mathrm{i}}^{2} \mathrm{f}_{\mathrm{i}} \mathrm{n}_{\mathrm{c}}}{\mathrm{x}_{\mathrm{c}}^{2}}}$ & $\sigma_{\mathrm{e}}=\sqrt{\frac{\sum \mathrm{x}_{\mathrm{i}}^{2} \mathrm{f}_{\mathrm{i}}}{\mathrm{n}_{\mathrm{e}}}-\mathrm{x}_{\mathrm{e}}^{2}}$ \\
$\sigma_{\mathrm{c}}=\sqrt{\frac{1171}{31}-5,90^{2}}$ & $\sigma_{\mathrm{e}}=\sqrt{\frac{2650}{36}-8,50^{2}}$ \\
$\sigma_{\mathrm{c}}=\sqrt{2,96}$ & $\sigma_{\mathrm{e}}=\sqrt{1,36}$ \\
$\sigma_{\mathrm{c}}=1,71$ & $\sigma_{\mathrm{e}}=1,16$ \\
\hline
\end{tabular}

Media aritmética - POST TEST

8.5

5.9

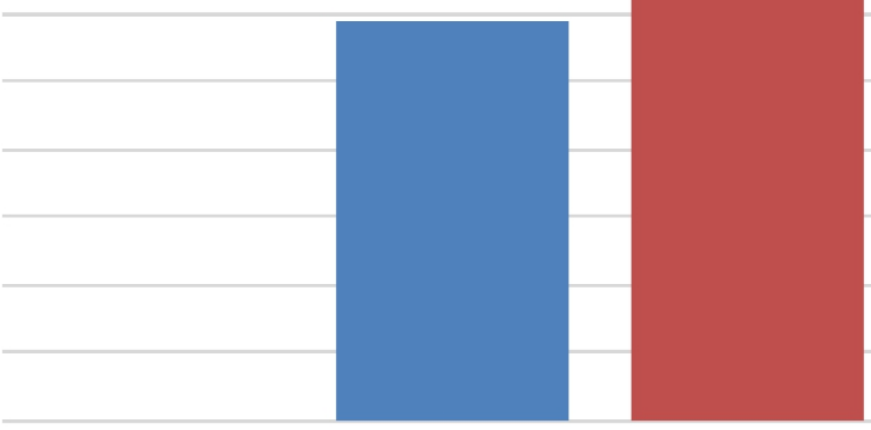

Gráfico 1. Media Aritmética POST Test (Grupo control vs Grupo experimental)

Se puede evidenciar claramente que, una vez implementada la herramienta tecnológica podcast, el promedio del grupo experimental es 
visiblemente mayor $(8,50)$ al del grupo de control $(5,90)$. La calificación promedio representa una mejora notable, ya que la misma, dentro de una escala de Valoración de Calificaciones, es MUY BUENA.

Tabla 4. Valoración de Calificaciones Obtenidas

\begin{tabular}{|c|c|}
\hline CALIFICACIÓN & VALORACIÓN \\
\hline 10 & Excelente \\
\hline 9 & Muy Bueno \\
\hline 8 & Bueno \\
\hline 7 & Aceptable \\
\hline$>6$ & Insuficiente \\
\hline
\end{tabular}

Elaborado por: Renato Ruiz López

Este resultado denota que el grupo experimental pudo obtener una mejor calificación en cuanto a la valoración de la comprensión auditiva, gracias a los beneficios aportados por la implementación del podcast como método de refuerzo en dicha destreza.

\section{Análisis y Prueba de Hipótesis}

A continuación, se determinará si las varianzas o variaciones esperadas con respecto a la media aritmética son las mismas para cada variable de estudio. Así se plantean las siguientes hipótesis:

Ho: Las varianzas entre tests son iguales

$$
\mathrm{x}_{\mathrm{c}}=\mathrm{x}_{\mathrm{c}}
$$

Ha: Las varianzas entre tests son distintas

Ho: Las varianzas entre grupos de control y experimental son iguales

$$
\mathrm{X}_{\mathrm{c}} \neq \mathrm{X}_{\mathrm{c}}
$$

Ha: Las varianzas entre grupos de control y experimental son distintas

Tabla 5. Evaluaciones Grupo de Control

\begin{tabular}{|l|c|c|}
\hline \multicolumn{1}{|c|}{$\begin{array}{c}\text { GRUPO DE } \\
\text { CONTROL }\end{array}$} & MEDIA & DESVIACIÓN ESTÁNDAR \\
\hline Pre Test & 5,81 & 1,88 \\
\hline Post Test & 5,90 & 1,71 \\
\hline Promedio General & 5,86 & 1,79 \\
\hline \multicolumn{3}{|c|}{$\begin{array}{c}\text { Fuente: Instrumentos de evaluación } \\
\text { Elaborado por: Renato Ruiz López }\end{array}$}
\end{tabular}

Tabla 6. Evaluaciones Grupo Experimental

\begin{tabular}{|c|c|c|}
\hline $\begin{array}{c}\text { GRUPO } \\
\text { EXPERIMENTAL }\end{array}$ & MEDIA & $\begin{array}{c}\text { DESVIACIÓN } \\
\text { ESTÁNDAR }\end{array}$ \\
\hline Pre Test & 5,50 & 1,62 \\
\hline Post Test & 8,50 & 1,16 \\
\hline Promedio General & 7,00 & 1,39 \\
\hline
\end{tabular}

Fuente: Instrumentos de evaluación

Elaborado por: Renato Ruiz López 


\section{Determinación de valores críticos y sus regiones de rechazo}

Mediante el cálculo de la prueba paramétrica $Z$ se rechaza la hipótesis nula si: $\mathrm{z}_{-} \mathrm{c}<-\mathrm{z}_{-} \mathrm{t}$ o también si $\mathrm{z}_{-} \mathrm{c}>\mathrm{z}_{-} \mathrm{t}$; $\llbracket \mathrm{z} \rrbracket \mathrm{t}_{-}=+/-1,96$ donde $z t$ es el valor teórico de $\bar{Z}$ para un nivel de significación del 5\%, $\alpha=0,05$; es decir que la investigación tendrá un $95 \%$ de confiabilidad; caso contrario se acepta la hipótesis de investigación con una de las dos alternativas.

Cálculos de la prueba paramétrica Z:

$$
x_{e}=7
$$

$x_{c}=5,86$

$\sigma_{\mathrm{e}}=1,39$

$\sigma_{\mathrm{c}}=1,79$

$$
\begin{aligned}
& n_{e}=36 \\
& n_{c}=31
\end{aligned}
$$

$$
z_{c}=\frac{x_{e}-x_{c}}{\sqrt{\frac{\sigma_{e}^{2}}{n_{e}}+\frac{\sigma_{c}^{2}}{n_{c}}}}
$$$$
z_{c}=\frac{7-5,8}{\sqrt{\frac{1,93}{36}+\frac{3,20}{31}}}
$$

$$
\mathrm{z}_{\mathrm{c}}=\frac{1,14}{0.396}
$$

$$
z_{c}=2,87
$$

\section{Toma de Decisión Estadística}

Al comparar el valor de $\mathrm{Z}$ calculado $(2,87)$ y el valor de $\mathrm{Z}$ teórico $(1,96)$, se puede observar que:

$$
\begin{gathered}
z_{-} \mathrm{c}>\mathrm{z}_{-} \mathrm{t} \\
2,87>1,96 .
\end{gathered}
$$

La regla de decisión es rechazar la hipótesis nula y aceptar la hipótesis alternativa, si el valor de $\mathrm{Z}$ calculado no queda en la región comprendida entre -1.96 y +1.96 . En caso contrario no se rechaza la hipótesis nula si $\mathrm{Z}$ queda entre $-1.96 \mathrm{y}+1.96$.

Por lo antes expuesto, es posible observar que $\mathrm{Z}$ calculado $=2,87$ se encuentra dentro de la zona de aceptación de la hipótesis de investigación, lo cual nos lleva a rechazar la hipótesis nula y a aceptar la hipótesis de investigación con su alternativa de incidencia positiva.

Por lo tanto, es deducible y comprobable que el grupo de estudiantes con el que se implementó el podcast tuvo una incidencia positiva por parte de esta herramienta tecnológica como método de refuerzo para la compresión auditiva del idioma inglés, a comparación del grupo que no contó condicha herramienta. 


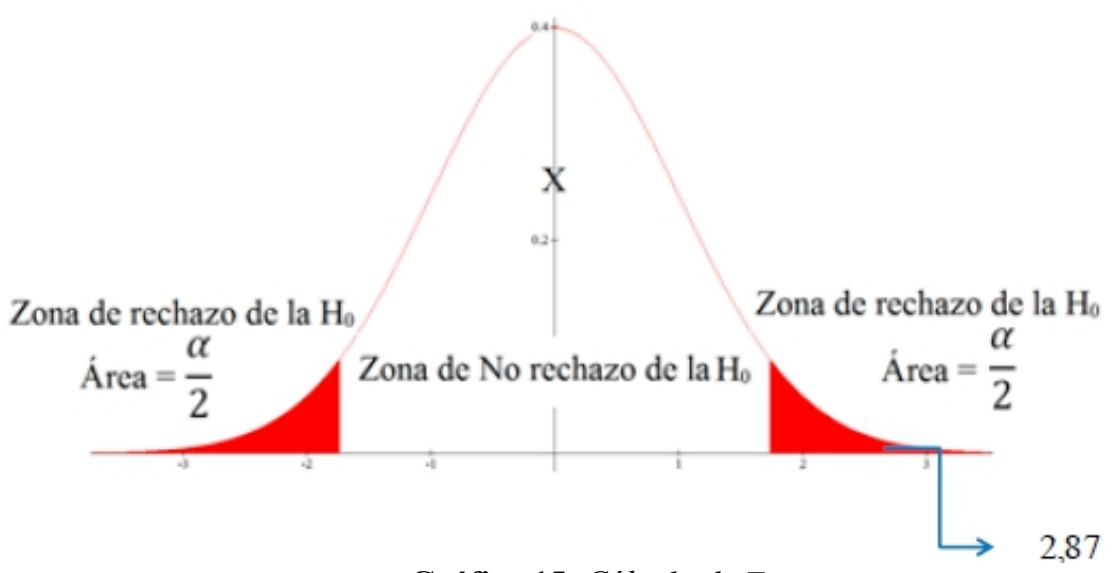

Gráfico 15. Cálculo de Z

\section{DISCUSIÓN}

Luego de la aplicación de la encuesta a los docentes del área de Lengua Extranjera de la Unidad Educativa Pedro Vicente Maldonado, y al observar los resultados obtenidos tanto en el pre test como en el post test del grupo experimental, es evidente que el trabajo en el aula de la compresión auditiva fue influenciado positivamente luego de haber aplicado el aprendizaje electrónico móvil a través del podcast) durante 8 semanas. Al comparar la media aritmética del pre test $(5,50)$ y del post test $(8,50)$, se logró un incremento en el puntaje de 3 puntos, lo cual equivale a un incremento en el rendimiento académico.

Como es lógico, en el grupo de control se observa que el promedio del pre test es 5,81 mientras que el del post test es 5,90. Esto demuestra que no existe una evolución en el rendimiento académico de los estudiantes en los que no se utilizó como método tecnológico el podcast. La media aritmética general también muestra que el grupo experimental supera al grupo de control, lo que permite afirmar que la hipótesis de investigación es cierta.

El cálculo del puntaje $Z$ arroja un valor de 2,87 ubicándose en la zona de aceptación de la hipótesis de investigación, lo cual permite rechazar la hipótesis nula y aceptar la hipótesis alterna, objeto de la presente investigación.

Es así que se puede afirmar mediante esta investigación que la puesta en marcha del aprendizaje electrónico móvil a través del podcast) incide positivamente en mejorar la comprensión auditiva en inglés de los estudiantes de Segundo de Bachillerato General Unificado "P" de la Unidad Educativa Pedro Vicente Maldonado durante el año lectivo 2016-2017.

\section{Conclusion}

El podcast es una herramienta tecnológica y didáctica muy útil dentro del proceso de enseñanza aprendizaje, específicamente en la comprensión 
auditiva de un idioma, ya que, al ser un archivo que se descarga inmediatamente y que puede abarcar múltiples contenidos, es capaz de convertirse en un maestro virtual para el estudiante actual que ya no se conforma con métodos tradicionales de enseñanza. Dentro de la presente investigación pudo comprobarse la efectividad de dicha herramienta luego de analizar los principios metodológicos dela misma, ya que al observar los resultados obtenidos tanto en el pre test (evaluación o diagnóstico), como en el post test aplicado al grupo experimental, es evidente el progreso en la compresión auditiva luego de la aplicación del podcast durante 8 semanas. Los estudiantes, en promedio, lograron un incremento en su puntaje de 3 puntos, lo que fundamenta la utilización del podcast como una efectiva herramienta de aprendizaje.

Antes de la implementación del aprendizaje electrónico móvil a través del podcast, la comprensión auditiva del idioma inglés de los estudiantes del Segundo Año de Bachillerato de la Unidad Educativa Pedro Vicente Maldonado era baja, resultados que cambiaron positivamente al implementar esta herramienta tecnológica ya que no sólo se logró una mejor compresión auditiva del idioma inglés en los estudiantes, sino también se desarrollaron varias destrezas en ellos, como por ejemplo: Mayor fluidez en el habla (speaking), mejor desenvolvimiento en clase (interacción), mayor capacidad de respuesta a las actividades propuestas (feedback), mejora e incremento del vocabulario y un notorio cambio de actitud en sus clases de inglés.

Para lograr una efectiva compresión auditiva del idioma inglés es necesario entonces aplicar métodos y técnicas que se acoplen a la realidad actual de los estudiantes, al entorno en el que se desenvuelven y a sus necesidades comunicacionales, razón por la cual el podcasting constituyó una técnica entretenida y multifuncional que estuvo acorde a la realidad estudiantil.

\section{RECOMENDACIONES}

- Adaptar el proceso de enseñanza - aprendizaje a métodos actuales y tecnológicos, que conminen a los estudiantes a tener mayor interés por la asignatura impartida, y sobre todo que, de una forma didáctica e interactiva, les ayuden a mejorar su rendimiento académico.

- Aprovechar todas las utilidades educativas del uso del podcast en el proceso de enseñanza aprendizaje, para lograr una mejor comprensión auditiva del idioma inglés en los estudiantes.

- Reforzar cada una de las destrezas del idioma inglés con una metodología específica, priorizando las necesidades de aprendizaje de los estudiantes para obtener mejores resultados, en concordancia con el grupo académico con el que trabaja el docente. 
- Utilizar todos los insumos tecnológicos y herramientas estudiantiles disponibles en las unidades educativas, ya que muchas veces están subutilizadas por los docentes y no se aprovechan al máximo sus bondades y ventajas tanto para el docente como para el estudiante. Es posible utilizar el internet como una herramienta divertida pero al mismo tiempo de ayuda para el aprendizaje continuo, sin barreras de tiempo ni espacio.

\section{References:}

1. Asociación Internacional de la escucha (1996).

2. Boquete, G. (2011). Las detrezas Orales.Alcalá: Universidad de Alcalá.

3. Boulos, M., Maramba, I. \& Wheeler, S. (2006). WIKIS, BLOGS AND PODCASTS: A NEW GENERATION OF WEB-BASED TOOLS FOR VIRTUAL COLLABORATIVE CLINICAL PRACTICE AND EDUCATION, BMC Medical Education, VOL. 6, No. 41;pp.1-8

4. Chamot, A.U. (1995). "Learning strategies and listening comprehension". San Diego. Págs. 13-30.

5. Chan, A. \& Lee, M. (2005). An mp3 a day keeps the worries awayexploring the use of podcasting to address preconceptions and alleviate pre-class anxiety amon gst undergraduate information technology students. http://www.csu.edu.au/division/studserv/sec/papers/chan.pdf)

6. Goss, B. (1982) "Listening as information processing", Communication Quarterly, Vol. 30, No4. Págs. 304-307

7. Kinshuk (2003). Adaptive Mobile Learning Technologies. Última visita: 18-01-17.

8. Martín Peris, E. (1996). Las actividades de aprendizaje en los manuales de ELE. Tesis doctoral. Disponible en su página personal.

9. Miller, M., \& Piller, M. (2005). Principal factors of an audio reading delivery mechanism - evaluating educational use of the iPod, Kommers, P. y Richards, G. (Eds.). Proceedings of World Conference on Educational Multimedia, Hypermedia and Telecommunications 2005. Chesapeake: AACE; pp. 260-267

10. Quinn, C. (2001). M-learning: Mobile, Wireless, in your pocket learning. Line Zine. Fall.

11. Sellas, T. (2011). “Tesis doctoral - La voz de la web 2.0”.Italia. 